\title{
Stückgutförderung von Mikroteilen mit der Schwingrinne
}

PROF. DR.-ING. RAINER BRUNS

DIPL.-WI.-ING. GUIDO GRAVENKÖTTER

INSTITUT FÜR KONSTRUKTIONS- UND FERTIGUNGSTECHNIK, PROFESSUR FÜR MASCHINENELEMENTE UND

TECHNISCHE LOGISTIK, HELMUT-SCHMIDT-UNIVERSITÄT HAMBURG

\section{Zusammenfassung}

Der vorliegende Beitrag greift das Auftreten von Größeneffekten auf, welche auf dem WGTL-Kolloquium 2006 vorgestellt worden sind und nun Gegenstand tiefer gehender Forschung sind. Dabei handelt es sich um einen Wechsel von Sekundäreffekten zu Primäreffekten, ausgelöst durch das Downscaling der Bauteildimensionen und somit eines veränderten Verhältnisses von der Masse zur Oberfläche.

Es werden erste Ergebnisse aus der Abschätzung der Kräfte vorgestellt, und die Auswirkungen dieser Kräfte auf den Fördervorgang diskutiert.

\section{Abstract}

This paper deals with scaling effects which have been discussed at the WGTL Colloquium 2006 and are now analysed in more detail. It describes the shift from secondary to primary effects due to the down scaling of construction components what leads to a changed mass-surface ratio.

First results of the force estimates are presented and the effects of these forces onto the conveying process are discussed.

\section{Einleitung}

Durch die zunehmende Verbreitung von Mikrosystemen und des somit bevorstehenden Übergangs zur Massenproduktion steigt die Notwendigkeit, die Zuführtechnik an die Besonderheiten einer Mikrofertigung anzupassen. Dabei kommt es zu physikalischen und wirtschaftlichen Größeneffekten, die in einer ersten Arbeit ausführlich beschrieben worden sind [Bruns06].

Schwingförderer stellen 80 \% der eingesetzten Zuführtechnik. Sie dienen der Förderung von unterschiedlichsten Stück- und Schüttgütern und sind gekennzeichnet durch:

+ einen einfachen Aufbau,

$+\quad$ eine hohe Betriebssicherheit und

+ einen geringen Wartungsaufwand.

Dem gegenüber stehen

- $\quad$ eine relativ hohe Geräuschentwicklung und

- $\quad$ kurze Förderlängen.

Das wesentliche Unterscheidungsmerkmal für Schwingförderer ist die Art des Antriebs (vgl. Abb. 1). Die häufigsten Antriebsarten sind Kurbeltrieb, Unwuchtantrieb und Elektromagnetantrieb [Wehmeier64]. Bei den Schwingrinnen überschreitet die vertikale Rinnenbeschleunigung den 

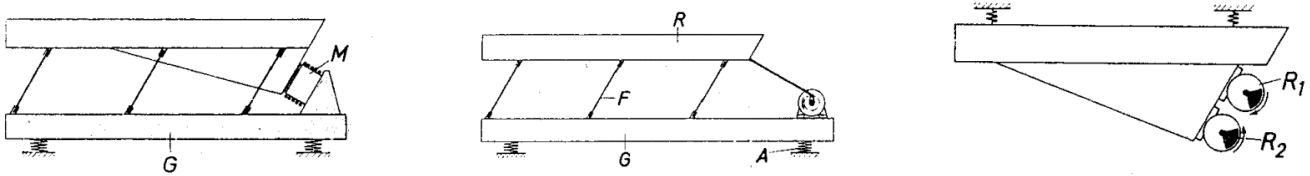

\section{Abbildung 1: Antriebsarten von Schwingrinnen; li. nach re.: Elektromagnetantrieb, Kurbeltrieb, Unwuchtantrieb}

Wert der Erdbeschleunigung und es setzt eine Kombination von kleinen Sprüngen (Mikrowurf) und kleinen Gleitbewegungen ein: das Fördergut hebt von der Rinne ab und bewegt sich in Förderrichtung. Erreicht die Rinnenbeschleunigung nicht die Erdbeschleunigung, d. h. bleibt das Fördergut immer in Kontakt mit dem Rinnenboden, dann setzen Gleitvorgänge ein, die durch unterschiedlich große Anpress- und Reibkräfte das Fördergut vorwärtsbewegen; nach diesem Prinzip fördern die Schüttelrutschen [Wehmeier61].

Eine spezielle Ausführung für die senkrechte Förderung stellen die Wendelschwingrinnen dar.

Der Einsatz der beschriebenen Zuführtechnik für die Förderung von Mikroteilen für die Mikrosystemtechnik erfordert eine genaue Analyse der auftretenden Größeneffekte. Denn die veränderten Verhältnisse von Oberflächen- zu Volumenkräften bei dem Downscaling der Bauteildimensionen führen dazu, dass physikalische Effekte in den Vordergrund treten, die vorher bedeutungslos waren. Dabei handelt es sich zum einen um Adhäsionskräfte, zum anderen aber auch um Strömungskräfte, die den Fördervorgang eines Mikroteils auf einer Schwingrinne beeinflussen. Aufgrund ihrer geringen Auswirkung spielten sie in der Makrowelt bisher nur eine untergeordnete Rolle. Diese Arbeit soll darauf hinweisen, dass die Vereinfachungen unzulässig sind, sobald die Dimensionen sich im Mikrobereich bewegen.

\section{Relevante Kräfte für die Förderbewegung}

Auf das zu fördernde Gut wirken Volumenkräfte, also Kräfte, die über das Volumen verteilt sind, und Flächenkräfte, die nur an der Oberfläche des Fördergutteilchens angreifen. Die einzelnen Kräfte und die Bedeutung hinsichtlich des Fördervorgangs (vgl. Abb.2) werden in diesem Kapitel näher erläutert.

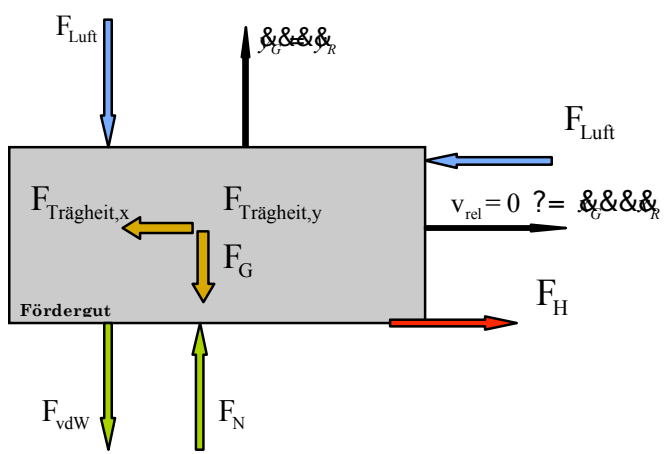

Abbildung 2: Freikörperbild eines Förderguts auf der Schwingrinne (Haftphase)

\subsection{Gewichtskraft FG}

Die Gewichtskraft wird durch die folgende Beziehung definiert:

$$
\mathrm{F}_{\mathrm{G}}=\mathrm{m} \cdot \mathrm{g}=\rho \mathrm{V} \cdot \mathrm{g} \text { Formel-Kapitel (nächstes) Abschnitt 2(2.1) }
$$


Sie ist also - neben der Erdbeschleunigung - nur vom Volumen und der Dichte eines Teilchens abhängig. Werden Länge, Breite und Höhe jeweils um ein Zehntel verkleinert, dann reduziert sich die Gewichtskraft um ein Tausendstel $\left(=1 / 10^{3}\right)$. Für den Fördergang hat die Gewichtskraft eine entscheidende Bedeutung. Sie bestimmt in der Kontaktphase (Fördergut und Rinne berühren sich) die Höhe der Anpresskräfte, und in der Flugphase (das Teil hebt vom Rinnenboden ab) die Teilchenbeschleunigung. Aufgrund ihrer Bedeutung für den Fördervorgang ist sie die wichtigste Bezugsgröße zu den Flächenkräften, die im Mikrobereich zur Geltung kommen. Die folgende Abbildung (Abb.3) veranschaulicht die schnellere Abnahme der Gewichtskraft gegenüber der Flächenkraft bei gleichmäßiger Verringerung aller drei Dimensionen:

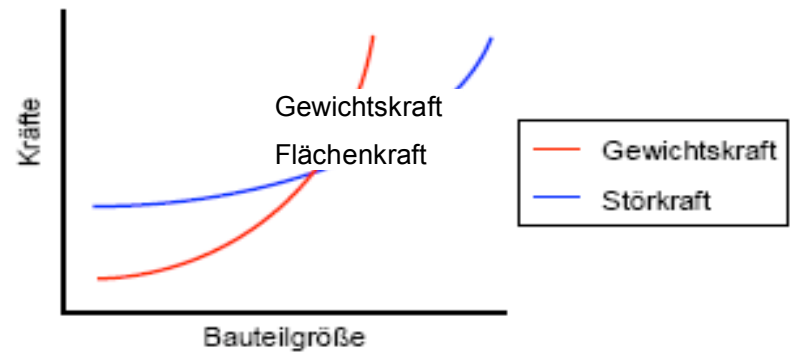

Abbildung 3: Abnahme der Volumenkraft gegenüber der Flächenkraft (Skizze)

\subsection{Massenträgheitskraft $\mathbf{F}_{\text {Trägheit,x/y }}$}

Soll ein Körper beschleunigt, abgebremst oder gedreht werden, so wirkt die Massenträgheit dieser Bewegung entgegen. Damit ist sie wie die Gewichtskraft ebenfalls von der Masse des Körpers, also von Dichte und Volumen abhängig. Für die translatorische Bewegung z. B. in x-Richtung gilt:

$$
\mathrm{m} \cdot \ddot{\mathrm{x}}=\sum_{\mathrm{i}} \mathrm{F}_{\mathrm{x}, \mathrm{i}}
$$

Die Schwingrinne muss das Fördergut gegen die Massenträgheit bewegen. Je schwerer ein Teilchen ist, desto größer muss die Anregungskraft der Rinne sein.

\subsection{Reibungskraft $\mathbf{F}_{H}$}

Die maximale Reibungskraft, die während der Haftphase zwischen Mikroteil und Rinne übertragen werden kann, wird durch die Normalkraft und den Haftbeiwert begrenzt. Die tatsächliche Haftkraft ist unbekannt.

$$
\left|\mathrm{F}_{\mathrm{H}}\right| £ ?=\mathrm{F}_{\mathrm{N}} \quad \mathrm{F}_{\mathrm{H}, \max }
$$

Die Normalkraft $\mathrm{F}_{\mathrm{N}}$ lässt sich nur indirekt aus den Gleichgewichtsbedingungen (vgl. Abb. 1) ermitteln:

$$
\mathrm{F}_{\mathrm{N}}=\mathrm{F}_{\mathrm{L}, \mathrm{y}}+\mathrm{m}\left(\mathrm{g} \quad \& \& \quad \mathrm{~F}_{\mathrm{vdW}}\right.
$$

Während der Aufwärtsbewegung wird die Normalkraft zusätzlich durch die Massenträgheit erhöht, in der Abwärtsbewegung nimmt sie ab, bis sie schließlich zu null wird, und das Teil sich von der Rinne löst und zur Wurfphase übergeht. Ein großer Reibbeiwert trägt dazu bei, dass das Teil während der Haftphase von der Rinne in Förderrichtung mitgenommen wird, was die Fördergeschwindigkeit erhöht. Dieses ist wichtig, da das Teil am Ende der Wurfphase wieder beschleunigt werden muss, um erneut aus der Haftphase in die nächste Wurfphase übergehen zu können.

\subsection{Adhäsionskraft (van-der-Waals-Kraft $\mathbf{F}_{v d w}$ )}

Als Adhäsion bezeichnet man das Aneinanderhaften zweier Stoffe. Liegen zwei extrem glatte Oberflächen aufeinander, so wirkt zwischen ihnen eine schwache Anziehungskraft, die umso größer ist, je dichter die Kontaktflächen aufeinander liegen. Mit zunehmendem Abstand nimmt sie jedoch sehr schnell ab. Die van-derWaals Kraft wird auf die Größe der Kontaktfläche bezogen und wird daher häufig in N/m² angegeben. Sie ist eine Flächenkraft. Auch die Werkstoffeigenschaften haben einen Einfluss auf die Größe der Adhäsionskraft. Je nach Werkstoffpaarung und umgebendem Medium werden sie zu einer Konstante zusammengefasst: der HAMAKER-Konstante. 
Die vdW-Bindungen und die dabei wirkenden vdW-Kräfte werden ohne Elektronenübergang oder Bildung von Elektronenpaaren gebildet. Sie beruhen nur auf asymmetrischen Verteilungen von positiven und negativen Ladungen innerhalb der Atome oder Moleküle. Ein polares Molekül mit einem Dipolmoment erzeugt nun eine Ladungstrennung in einem sonst nichtpolaren Molekül (Influenz), was zu einer gegenseitigen elektrostatischen Anziehung der Moleküle führt; man spricht daher auch von Dipol induzierten Bindungen [Giancoli06, Shackelford05].

Die vdW-Bindungen gehören daher zu den schwachen Bindungen $(0.04 \mathrm{eV}$ bis $0.3 \mathrm{eV})$ und treten nur dann in Erscheinung, wenn sich zwei Atome oder Moleküle im sehr geringen Abstand $r$ zueinander befinden. Für die vdW-Kraft gilt folgender Zusammenhang:

$$
\mathrm{F}_{\mathrm{vdW}} \frac{1}{\mathrm{r}^{7}}
$$

Da sich die vdW-Kräfte bei einer Fläche addieren $\left(\mathrm{F}_{\mathrm{PP}}\right)$, nehmen sie dort allerdings weniger schnell mit dem Plattenabstand $d$ ab und folgen einem anderen Gesetz:

$$
\mathrm{F}_{\mathrm{PP}} \frac{1}{\mathrm{~d}^{3}}
$$

Diese Theorie wurde von Casimir und Polder weiterentwickelt („retarded van-der-Waals-Forces“). Sie weisen darauf hin, dass es eine endliche Zeitspanne dauert, bis das elektrische Feld des ersten Atoms das zweite Atom erreicht, währenddessen sich das Feld im ersten Atom schon wieder geändert hat. Ein Ergebnis ihrer Berechnungen war, dass die Anziehungskraft um den Faktor 1/r abgeschwächt wird $\left(\mathrm{F}_{\mathrm{PP}} \sim 1 / \mathrm{d}^{4}\right)$.

Einen makroskopischen Ansatz verfolgte 1956 die Lifshitz-Theorie, die als Grundlage für die weitere Abschätzung dient. Lifshitz zeigte, dass die Wechselwirkungsenergie lediglich von der makroskopischen Materialkonstante $\varepsilon_{0}$, also der statischen Dielektrizitätskonstante des Mediums, abhängig ist $\left(\mathrm{B}_{132}=\right.$ HamakerKonstante für retarded Forces, $\rho=0,35 \ldots 1$, c=Lichtgeschwindigkeit) [Maugis00, Parsegian06]:

$$
\begin{aligned}
\mathrm{F}_{\mathrm{PP}}(\mathrm{d}) & =\frac{\pi^{2} \hbar \mathrm{c}}{240 \mathrm{~d}^{4}} \frac{1}{\sqrt{\varepsilon_{30}}}\left(\frac{\varepsilon_{10}-\varepsilon_{30}}{\varepsilon_{10}+\varepsilon_{30}}\right)\left(\frac{\varepsilon_{20}-\varepsilon_{30}}{\varepsilon_{20}+\varepsilon_{30}}\right) \varphi\left(\varepsilon_{10}, \varepsilon_{20}, \varepsilon_{30}\right) \\
& =\frac{\mathrm{B}_{132}}{\mathrm{~d}^{4}}\left[\frac{\mathrm{N}}{\mathrm{m}^{2}}\right]
\end{aligned}
$$

Häufig werden Teile der Mikrosystemtechnik mit speziellen Verfahren wie dem LIGA-Verfahren hergestellt. Diese Teile basieren auf einem Siliziumwerkstoff und haben sehr geringe Oberflächenrauigkeiten. Ein weiteres Beispiel sind Bauteile, die mit dem PVD-Verfahren metallisch beschichtet (,gesputtert“) werden. Hierbei werden ebenfalls Oberflächenrauigkeiten im Nano-Bereich hergestellt. Die Handhabung dieser Teile (Abb.4) mit der konventionellen Materialflusstechnik wird daher aufgrund der beschriebenen Adhäsionskräfte und des nur geringen Gewichts sehr schwierig.
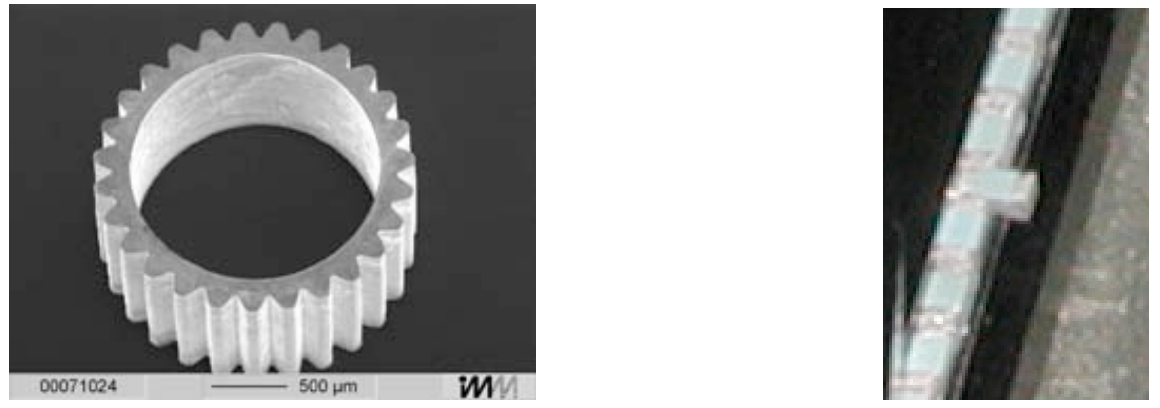

Abbildung 4: links: ein mit dem LIGA-Verfahren hergestelltes Zahnrad (Quelle: IMM); rechts: SMDWiderstände auf einer Wendelschwingrinne (1 $\mathrm{mm}$ x $0.5 \mathrm{~mm}$ x $0.35 \mathrm{~mm}$, Quelle: Vishay-Beyschlag)

Mit Hilfe von Gleichungen (2.7) kann eine Abschätzung für zwei identische, metallische Platten (d.h. $\varepsilon_{10,20} \rightarrow \infty$ ) die aufeinander liegen und nur durch die Oberflächenrauigkeit getrennt werden) erfolgen. Abbildung 5 zeigt die Gewichtskraft, die über den Abstand konstant bleibt, und die vdW-Kraft, die immer größer wird, je geringer die Oberflächenrauhigkeit ist (d. h. je glatter die Platten sind): 


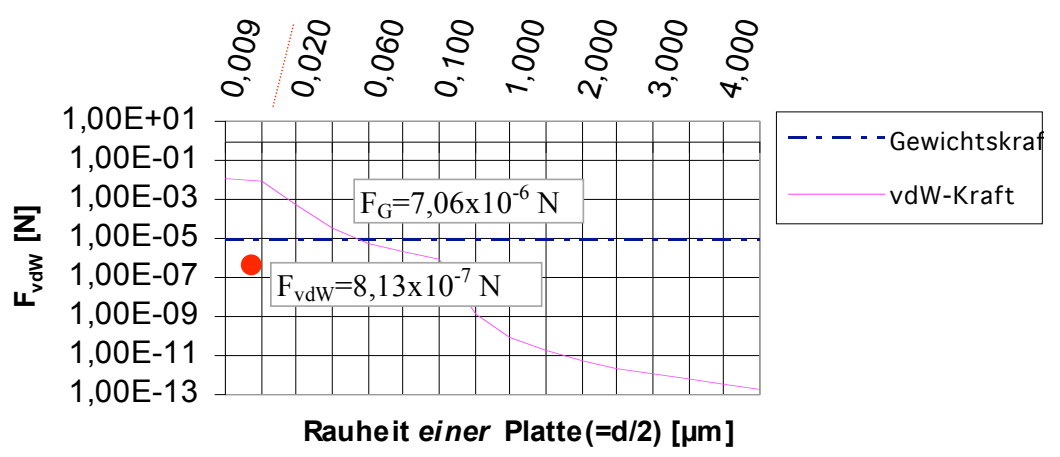

Abbildung 5: Abschätzung der Adhäsionskraft durch Veränderung der Oberflächenrauhigkeit

Es wird deutlich, dass die vdW-Kraft zwischen zwei Oberflächen mit einer Oberflächenrauhigkeit von $0,1 \mu \mathrm{m}$, d.h. bei einem Plattenabstand von $\mathrm{d}=0,2 \mu \mathrm{m}, 10 \%$ der Gewichtskraft annimmt. Für noch geringere Abstände wächst das Verhältnis Adhäsions- zu Gewichtskraft noch stärker an. Die Bauteilhöhe hat keinen Einfluss auf die Adhäsionskraft sondern nur auf die Gewichtskraft. Wird nur die Bauteilhöhe - bei gleich bleibender Kontaktfläche - verkleinert, dann verringert sich anteilsmäßig nur die Gewichtskraft, bzw. das Verhältnis von Adhäsions- zur Gewichtskraft nimmt um den Kehrwert zu. Eine Verringerung der Bauteilhöhe um den Faktor 2 hat beispielsweise eine Verdopplung des Kräfteverhältnisses zur Folge. Im Vergleich zur Kontaktfläche besonders dünne Teilchen, neigen daher eher zu Adhäsionserscheinungen.

Die zusätzliche Adhäsionskraft erhöht die Normalkraft, was dazu führt, dass höhere Reibungskräfte übertragen werden können und sich der Abhebezeitpunkt auf einen späteren Zeitpunkt verschiebt. Detaillierte Erkenntnisse zu den Auswirkungen liegen noch nicht vor und müssen erst noch durch weitere Versuche gewonnen werden.

\subsection{Strömungskraft $\mathbf{F}_{L}$}

Ein weiterer wesentlicher Effekt, der beim Downscaling in Erscheinung tritt, ist der Einfluss von Strömungskräften, die durch den Luftwiderstand, den das Mikroteil bietet, hervorgerufen werden. Da die Rinne gleichzeitig eine Bewegung in vertikale und horizontale Richtung durchführt, kommt es auf der einen Seite zu vertikalen Strömungskräften $F_{L y}$ - normal zur erzwungenen Rinnenbewegung - die das Abheben erschweren, und während des Falls die Bewegungen abbremsen; und auf der anderen Seite zu horizontalen Strömungskräften $F_{L x}$ - tangential zur Rinnenoberfläche - die die Parallelbewegung in Förderrichtung durch den Luftwiderstand hemmen. Durch diese Strömungseinflüsse schlägt das Mikroteil erheblich früher wieder auf der Rinne auf (Abb.6). Der Aufprallzeitpunkt verlagert sich nach vorn und in Abhängigkeit der Rinnenposition verringert sich dadurch die Relativgeschwindigkeit zwischen Teil und Rinne, und damit auch die Aufprallenergie. Ob es sich um eine laminare oder turbulente Strömung handelt, wird noch weiter untersucht.
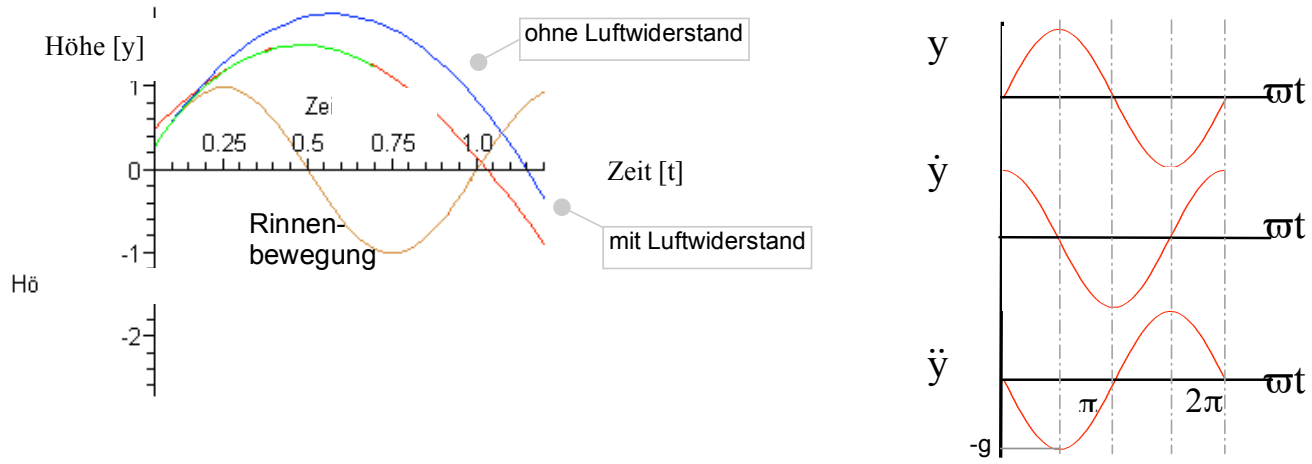

Abbildung 6: links: Auswirkung des Luftwiderstands auf die vertikale Teilchenbewegung; rechts: Geschwindigkeit und Beschleunigung 
Im Makrobereich wurden diese Einflüsse nicht beachtet, und fanden allenfalls bei der Berechnung der Schüttgutförderung, im Hinblick auf die Luftkissenbildung und der damit eingeschränkten Förderhöhe und Fördergeschwindigkeit, eine Berücksichtigung [Böttcher58].

Die Strömungskraft ist von der Anströmungsgeschwindigkeit $\dot{y}_{G}$, der Querschnittsfläche $A$ senkrecht zur Anströmungsgeschwindigkeit, dem $\mathbf{c}_{\mathbf{W}}$-Wert der von der Geometrie der angeströmten Fläche bestimmt wird und

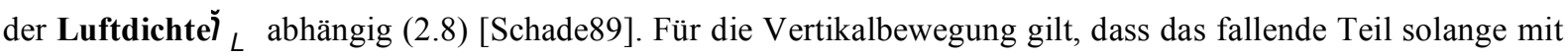
der Erdanziehung beschleunigt wird, bis der Luftwiderstand und die Gewichtskraft im Gleichgewicht sind. Ab dann fällt es nur noch mit einer konstanten Geschwindigkeit.

$$
\mathrm{F}_{\mathrm{L}}=\frac{1}{2} \rho_{\mathrm{L}} \mathrm{c}_{\mathrm{w}, \mathrm{y}} \cdot \mathrm{A} \cdot \sqrt{\dot{\mathrm{y}}_{\mathrm{G}}^{2}} \cdot \dot{\mathrm{y}}_{\mathrm{G}}
$$

Für die Anströmungsgeschwindigkeit ist allein die Geschwindigkeit der Rinne zum Zeitpunkt des Abhebens von Bedeutung, und diese wird nur durch Amplitude und Frequenz der Schwingrinne bestimmt. Allgemein gilt für die Bewegung der Rinne (Amplitude $\hat{y}, \omega=2 \pi f$ ):

$$
\begin{aligned}
& \mathrm{y}_{\mathrm{R}}(\mathrm{t})=\hat{\mathrm{y}} \cdot \cos (\omega \mathrm{t}) \\
& \dot{\mathrm{y}}_{\mathrm{R}}(\mathrm{t})=\hat{\mathrm{y}} \omega \cdot \cos (\omega \mathrm{t}) \\
& \ddot{\mathrm{y}}_{\mathrm{R}}(\mathrm{t})=-\hat{\mathrm{y}} \omega^{2} \cdot \sin (\omega \mathrm{t})
\end{aligned}
$$

Trägt man die Strömungskraft für die rein vertikale Bewegung über die Gewichtskraft prozentual auf, und variiert die Bauteilhöhe $h$, dann ergibt sich folgender Verlauf (Abb.7):

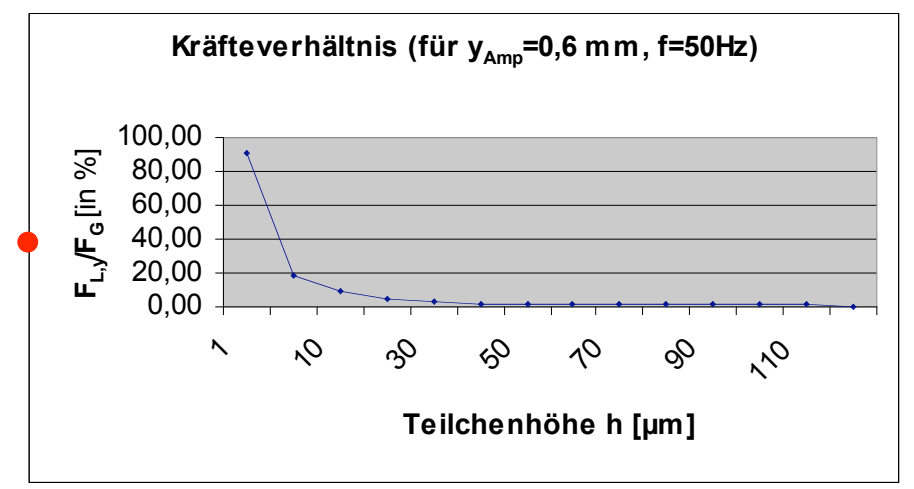

Abbildung 7: Abschätzung der vertikalen Strömungskraft zur Gewichtskraft bei Variation der Teilchenhöhe

Durch die Verringerung der Bauteilhöhe sinkt die Gewichtskraft des Mikroteils, und die Widerstandskraft gewinnt an Bedeutung. Im obigen Beispiel wird deutlich, dass bei einer Bauteilhöhe von ca. $10 \mu \mathrm{m}$, der Einfluss des Luftwiderstandes etwa $10 \%$ beträgt und damit bemerkbar wird. Mit zunehmender Bauteilhöhe nimmt das Verhältnis rasch ab. Diese hohe Empfindlichkeit sehr dünner Teilchen gegenüber einer Luftströmung muss bei der Gestaltung der Förderumgebung, durch entsprechenden Schutz vor Luftströmungen, Rechnung getragen werden.

Der Verlauf des Diagramms ist maßgeblich von den Parameterwerten der Schwingrinne (Amplitude und Frequenz) abhängig, da sie den Geschwindigkeitsverlauf der Rinnenbewegung beschreiben, welche quadratisch in die Berechnung der Strömungskraft (2.8) eingeht.

Für die Betrachtung der Vertikalbewegung ist nur eine Dimension ausschlaggebend, nämlich die Bauteilhöhe. Denn die Grundfläche $A$ ist sowohl in der Strömungskraft als auch der Gewichtskraft enthalten, und eine Veränderung wäre somit ohne Wirkung. Die Verkleinerung der Höhe des Mikroteils hat aber zur Folge, dass sich seine Gewichtskraft (bei konstanter vertikaler Strömungskraft) verringert. Wie bei der Adhäsionskraft ändert sich auch hier das Verhältnis von Strömungs- zu Gewichtskraft mit dem Kehrwert der Veränderung der Bauteilhöhe. Eine Halbierung der Bauteilhöhe hat auch hier eine Verdopplung des Kräfteverhältnisses zur Folge. Für die Betrachtung der Parallelbewegung gelten die Aussagen analog für die Variation der Bauteillänge anstatt der Bauteilhöhe. 
Die Oberflächenrauhigkeit des Fördergutes ist - anders als bei der Adhäsionskraft - für die Strömungskraft nicht von Bedeutung. Ebenso wenig haben die physikalischen Eigenschaften des Werkstoffs einen Einfluss auf die Höhe des Luftwiderstands.

\section{Zusammenfassung und Ausblick}

Schwingförderer haben ihren festen Platz in der Zuführtechnik für Fertigung, Montage und andere Aufgaben. Die zunehmende Verbreitung von Mikrosystemen und die Fortschritte in der Miniaturisierung lassen vermuten, dass die zu fördernden Bauteile immer kleinere Dimensionen annehmen werden, womit aufgrund von Größeneffekten neue Anforderungen an die Zuführtechnik entstehen. Die Größeneffekte, Adhäsionskraft und Strömungskraft im Vergleich zur Gewichtskraft, wurden ausführlich in Kapitel 2 hergeleitet und hinsichtlich des Fördervorgangs eines Mikroteilchens auf einer Schwingrinne quantifiziert. Dabei ist deutlich geworden, dass beide Effekte eine Rolle spielen (etwa 10 \%), wenn das zu fördernde Gut in den Mikrobereich herunterskaliert wird.

Doch die Größeneffekte sind nicht nur von den Bauteildimensionen abhängig, sondern auch von den physikalischen Eigenschaften der Kontaktflächen und den Betriebseinstellungen bezüglich Amplitude und Frequenz der Schwingrinne. Dieses ist für die Planung der Versuchsreihen relevant.

Die Entwicklung eines Modells zur Berücksichtigung der Größeneffekte beim Fördervorgang ist Ziel der Forschungsarbeit. Dazu werden die vertikalen und horizontalen Bewegungsgleichungen, unter Zuhilfenahme dimensionsloser Größen, aufgestellt. Eine anschließende Modellverifizierung wird dann mithilfe einer Hochgeschwindigkeitskamera im Labor MTL durchgeführt.

Aus den Forschungsergebnissen sollen zukünftige Anforderungen an die Materialflusstechnik abgeleitet werden, die sie für einen Einsatz in der Mikrosystemtechnik befähigen.

\section{Literatur}

[Böttcher58]

[Bruns06]

[Giancoli06]

[Maugis00]

[Parsegian06]

[Schade89]

[Shackelford05]

[Wehmeier61]

[Wehmeier64]
Böttcher, S.: Beitrag zur Klärung der Gutbewegung auf Schwingrinnen (II), (III), Fördern und Heben, 4 (1958), S.235-240, 5 (1958), S.307-315

Bruns, R., Gravenkötter, G.: Größeneffekte in der Mikrologistik. Logistics-Journal ISSN 1860-5923, 11(2006)

http://www.elogistics-journal.de/archiv/2006/november/634 (Datum des letzten Zugriffs: 09.02.2007)

Giancoli, D.: Physik. Pearson Studium ISBN 978-3-8273-7157-7 (2006)

Maugis, D.: Contact, adhesion and rupture of elastic solids. Springer Verlag, ISBN 3-540-66113-1 (2000)

Parsegian, V.A.: van-der-Waals-Forces. Cambridge University Press ISBN 978-0521-54778-9 (2006)

Schade, H., Kunz, E.: Strömungslehre. Gruyter, de ISBN 978-3110118735 (2000)

Shackelford, J.: Werkstofftechnologie für Ingenieure, Pearson Studium ISBN 38273-7159-7 (2005)

Wehmeier, K.H.: Untersuchungen zum Fördervorgang auf Schwingrinnen (1. Teil), Fördern und Heben 11 (1961),S.317-327

Wehmeier, K.H.: Schwingförderrinnen - Eine Systematik der Bauformen und ihre Eigenarten, Fördern und Heben, 3 (1964), S.155-161 